\title{
Quick Quasi-TEM Analysis of Multiconductor Transmission Lines with Rectangular Cross Section
}

\author{
Joaquín Bernal, Francisco Medina, and Manuel Horno, Member, IEEE
}

\begin{abstract}
This paper presents an efficient and accurate procedure for computing the quasi-static matrix parameters $([\mathrm{C}],[\mathrm{L}]$, $[G]$, and $[R]$ ) of rectangular-shaped conductors embedded in a multilayered dielectric medium over an infinite ground plane. An additional top ground plane can also be considered. The problem is formulated in terms of the space-domain integral equation for the free-charge distribution on the slab conductor surfaces. The spatial Green's function is computed from its spectral counterpart using system identification techniques [Prony's method or matrix pencil method (MPM)]. The integral equation is solved by means of a Galerkin scheme employing entire domain basis functions. This results in a small matrix size. In addition, the quasi-analytical evaluation of the entries of the Galerkin matrix leads to a very efficient and accurate computer code. A detailed study on the convergence and accuracy of the method has been included.
\end{abstract}

Index Terms-Losses, microstrip, multiconductor transmission lines, quasi-TEM analysis, thick conductors.

\section{INTRODUCTION}

A LARGE amount of papers have been devoted to the analysis of planar transmission lines throughout the last three decades. Most of the published work assumes negligible metallization thickness. This approximation is good enough for many practical situations, and permits the simplification and efficient use of the analysis ad hoc mathematical techniques. However, during the last few years many authors have paid attention to the problem of accounting for the nonzero metallization thickness. Apart from the aim of increasing accuracy, this interest comes from the necessity of analyzing the electrical behavior of the relatively thick strips employed in monolithic microwave circuits and high-speed digital circuits. In these cases, metallization thicknesses and strip widths are in the same order of magnitude. Therefore, the former can no longer be neglected. The strip thickness must also be considered whenever tight edge coupling is present, even though relatively wide strips are under consideration. Moreover, the computation of ohmic conductor losses requires explicit accountability for the strip thickness.

A number of authors have analyzed the effects of the metallization thickness by using a full-wave analysis, but in

Manuscript received October 4, 1996; revised March 25, 1997. This work was supported by the DGICYT, Spain, under Project TIC95-0447. The work of J. Bernal was supported by a grant from Junta de Andalucia (Spain).

The authors are with the Grupo de Microondas, Departamento de Electrónica y Electromagnetismo, Facultad de Física, Universidad de Sevilla, 41012 Seville, Spain.

Publisher Item Identifier S 0018-9480(97)06066-3. this paper we are only interested in quasi-static approaches. This is because of its comparative simplicity, which makes it useful to develop quick microstrip solvers. Full-wave methods, except when applied to zero-thickness printed lines, usually demand a lot of computer time. Even when a quasiTEM approach is used, accounting for strip-thickness effects precludes, in principle, the use of some very efficient analytical techniques developed for zero-thickness planar structures [1]-[3]. In principle, one might use some type of purely numerical approach, such as the finite-difference [4] or finiteelement techniques [5]-[7]. However, even though significant improvements have been introduced in the formulation of those methods, they are more appropriate when dealing with complicated geometries which cannot be analyzed with lesser computer resource-demanding techniques. A hybrid numerical/analytical method - the method of lines-has been recently applied to the analysis of coupled microstrips with finite thickness [8]. Nevertheless, solutions based on integralequation formulations seem to be well suited for most practical cases if the goal is to get high accuracy with low computational cost. Thus, arbitrary-section coupled conductors embedded in a layered medium have been analyzed in [9] by means of an integral-equation technique based on the free-space Green's function. This method was generalized in [10] to account for nonlayered dielectrics. Important numerical improvements on this technique have recently been reported in [11]. Other authors prefer to use a dielectric Green's function when dealing with layered dielectric substrates, since the number of unknowns is drastically reduced in this way [12]-[15]. The last five papers stressed the analytical preprocessing of the computations so as to enhance both accuracy and computational speed. Some other authors have reported different techniques to develop quick computational tools for the quasi-TEM analysis of particular microstrip structures with nonnegligible metallization thickness [16]-[19]. This paper fits into this research line.

In this paper, we propose a new method that combines the advantages of different formulations in order to build up a quick quasi-static computer solver for rectangular-shaped conductors embedded in a multilayered dielectric medium. The method is based on solving the space-domain integral equation for the free-charge distribution on the conducting slabs. The appropriate space-domain Green's function (the kernel) is conveniently obtained from the spectral one by means of system identification techniques (using the complex 


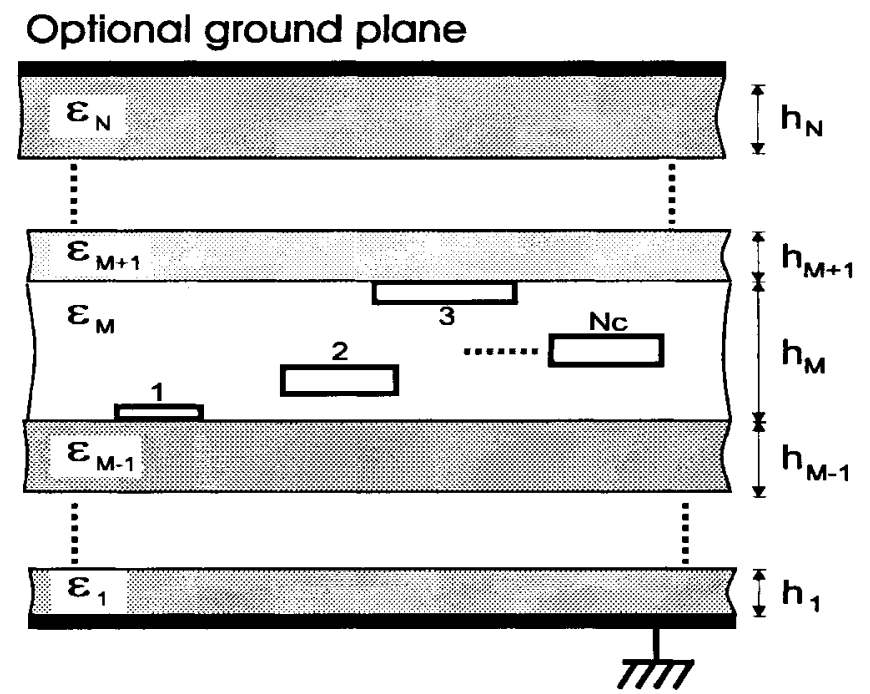

Fig. 1. The multiconductor transmission line studied in this paper. It consists of $N_{c}$ rectangular conducting slabs embedded in the $M$ th layer of a $N$-layers dielectric medium.

images concept [20]). Entire domain basis functions are used in a Galerkin scheme with the aim of keeping the size of the final Galerkin matrix small. In addition, the computation of the elements of this matrix is carried out in a very efficient way by using suitable numerical quadratures and closed-form integration. Putting together all these elements leads to an efficient and accurate computer code which is suitable for quick computations even on a PC platform.

\section{Statement of the Problem}

Consider a multiconductor transmission line such as the one shown in Fig. 1. An arbitrary number $N_{c}$ of rectangularshaped conductors are embedded inside the $M$ th dielectric layer of a multilayered ( $N$ layers) dielectric medium. A bottom ground plane is always present, while the top ground plane is optional. Our main purpose is to compute, in an efficient and accurate way, the per unit length complex capacitance matrix of the multiconductor system (i.e., the capacitance [C] and conductance $[\mathrm{G}]$ matrices). As is well known, the computation of the per unit length inductance matrix $[\mathrm{L}]$ reduces to the evaluation of $[\mathrm{C}]$ for the same structure without dielectrics. Finally, by using the Wheeler's incremental inductance rule, we can obtain the resistance matrix of the multiconductor system [R] from [L], as shown in [21].

As stated in the Section I, there exists a wide variety of techniques to compute the capacitance matrices of multiconductor transmission systems. We have chosen to solve the integral equation for the surface charge distribution on the rectangular conductors. This means that an appropriate Green's function accounting for the multiple boundaries and the bottom (and top) ground plates has to be computed. The spectral-domain version of such a Green's function can be easily obtained owing to the layered geometry of the dielectric region (see, for instance, the transverse transmission line (TTL) method reported in [22] or the method in [23]). If source and field points are inside the $M$ th layer, the spectral Green's function can be written in the following form:

$$
\begin{aligned}
\tilde{G}\left(\alpha \mid y, y^{\prime}\right)= & \frac{1}{2 \alpha \varepsilon_{0} \varepsilon_{M}\left(1-\Gamma_{L} \Gamma_{U} e^{\left.-2 \alpha h_{M}\right)}\right.}\left[\Gamma_{L} e^{-\alpha\left(y+y^{\prime}\right)}\right. \\
& +e^{-\alpha\left|y-y^{\prime}\right|}+\Gamma_{L} \Gamma_{U} e^{\alpha\left(\left|y-y^{\prime}\right|-2 h_{M}\right)} \\
& \left.+\Gamma_{U} e^{\alpha\left(y+y^{\prime}-2 h_{M}\right)}\right]
\end{aligned}
$$

$\Gamma_{L, U}$ in (1) stand for the reflection coefficients seen from the lower $(L)$ and upper $(U)$ surfaces bounding the $M$ th layer, provided we are using the equivalent transmission-line model to compute the spectral Green's function [22]. Of course, $\Gamma_{L, U}$ are known closed-form functions of the Fourier variable $\alpha$ and of the permittivities and thicknesses of the dielectric layers below $(L)$ or above $(U)$ the $M$ th one. In order to recover the $x$-dependence of the space-domain Green's function, a Fourier transform inversion has to be carried out:

$$
G\left(x, x^{\prime} \mid y, y^{\prime}\right)=\frac{1}{2 \pi} \int_{-\infty}^{\infty} d \alpha \tilde{G}\left(\alpha \mid y, y^{\prime}\right) e^{j \alpha\left(x-x^{\prime}\right)} .
$$

Apart from some particular cases, the inverse Fourier transform (2) cannot be performed in closed form. However, the $\alpha$-dependent functions appearing in (1) as multiplicative factors of the exponential terms could be written as a finite sum of complex exponentials. In this way, we could apply the ideas reported in [20] so as to get a very close approximation to the space-domain Green's function. This point will be discussed in Section III. Once the Green's function is known, we can solve the integral equation for the free-charge distribution by using, for instance, the Galerkin method. In Section IV, we will give details on the type of basis functions used in this paper and on the techniques applied in order to speed up computations. From the charge on the conducting slabs, we obtain the capacitance matrix. If the electrical permittivities of the dielectric layers are complex (dielectric losses), the elements of this matrix will be complex. Their imaginary parts give the elements of [G]. On the other hand, $[\mathrm{L}]$ is the inverse of the capacitance matrix for the structure without dielectrics over $c^{2}(c$ is the speed of light in vacuo). Finally, under strong skin-effect operation, we can compute the per-unit-length resistance matrix $[R]$, from [L] by using the extension to a multiconductor transmissionline system of the Wheeler's rule [21]. Therefore, with an accurate method to compute complex capacitance matrices, we will be able to obtain all the quasi-static matrix parameters characterizing our lossy multiconductor system (including the [R] matrix, provided the skin effect on metals is strong).

\section{Computation of the SPACE-Domain GREEN'S FUNCTION}

Following the guidelines in [20], the space-domain Green's function can be usually expressed as a short summation of terms which can be easily computed from its spectral-domain version. The spectral Green's function is known in closed form for a layered structure. The basic equation underlying Chow's procedure is the following relationship between spectral and spatial functions:

$$
\mathcal{F}\left[-\frac{1}{2 \pi} \ln \left(\left(x-x^{\prime}\right)^{2}+u^{2}\right)\right]=\frac{e^{-\alpha u}}{\alpha}
$$


where $\mathcal{F}$ stands for Fourier transform and $u$ is a generalized complex distance. If we can write (1) as a sum of terms such as the ones on the right-hand side (RHS) of (3), it is clear that we will have the space-domain Green's function of our problem. This can be done almost in a straightforward way by expanding the terms by multiplying the exponentials in (1) as a sum of complex exponentials. The arguments of the exponentials and the coefficients of the expansion can be computed by using spectral estimation methods. In this paper, we have used and compared the Prony's method employed in [20] and the MPM, as reported in [24].

A caution should be exerted when we are considering structures having a top ground plane in addition to the bottom one. In such a case, the spectral functions to be approximated are singular at the point $\alpha=0$. This behavior comes from the following factor:

$$
\frac{1}{1-\Gamma_{L} \Gamma_{U} e^{-2 \alpha h_{M}}} \approx \frac{1}{2 \alpha H[1+\alpha(2 d-H)]}, \quad \alpha \rightarrow 0
$$

where $H=\varepsilon_{M} \sum_{i=1}^{N}\left(h_{i} / \varepsilon_{i}\right)$ and $d=\varepsilon_{M} \sum_{i=1}^{M-1}\left(h_{i} / \varepsilon_{i}\right)$. A homogeneous structure with relative permittivity $\varepsilon_{M}$ having two ground planes separated by a distance $H$ has a spectral Green's function that can be expressed as follows:

$$
\begin{aligned}
\tilde{G}_{h}\left(\alpha \mid y, y^{\prime}\right)= & \frac{1}{2 \alpha \varepsilon_{0} \varepsilon_{M}\left(e^{-2 \alpha H}-1\right)}\left[e^{-\alpha\left(y+y^{\prime}\right)}\right. \\
& \left.-e^{-\alpha\left|y-y^{\prime}\right|}-e^{\alpha\left(\left|y-y^{\prime}\right|-2 H\right)}+e^{\alpha\left(y+y^{\prime}-2 H\right)}\right] .
\end{aligned}
$$

The Green's function in (5) and the original one have the same behavior around the $\alpha=0$ point. In addition, the space-domain Green's function for the homogeneous structure $G_{h}\left(x, y \mid x^{\prime}, y^{\prime}\right)$ is known in closed form:

$$
\begin{aligned}
G_{h}\left(x, y \mid x^{\prime}, y^{\prime}\right) \\
=\frac{1}{4 \pi \varepsilon_{0} \varepsilon_{M}} \\
\quad \times \ln \left\{\frac{\cosh \left[\pi\left|x-x^{\prime}\right| / H\right]-\cos \left[\pi\left(y+y^{\prime}\right) / H\right]}{\cosh \left[\pi\left|x-x^{\prime}\right| / H\right]-\cos \left[\pi\left(y-y^{\prime}\right) / H\right]}\right\} .
\end{aligned}
$$

Expression (6) can be considered as the first contribution to the complete Green's function of the layered structure. The additional terms are expressed in the spectral domain as the difference between (1) and (5). The terms of this spectral function have no singularities at $\alpha=0$ and can now be treated without problems.

In brief and after some algebra, the spectral-domain Green's function for the layered structure can be written in the following useful form:

$$
\begin{aligned}
& \widetilde{G}\left(\alpha \mid y, y^{\prime}\right) \\
& =\frac{1}{2 \alpha \varepsilon_{0} \varepsilon_{M}}\left\{e^{-\alpha\left|y-y^{\prime}\right|}+K e^{-\alpha\left(y+y^{\prime}\right)}+A_{1} e^{-\alpha\left(y+y^{\prime}\right)}\right. \\
& \left.+A_{2}\left(e^{-\alpha\left|y-y^{\prime}\right|}+e^{\alpha\left|y-y^{\prime}\right|}\right)+A_{3} e^{\alpha\left(y+y^{\prime}\right)}\right\} \\
& +\delta\left[\tilde{G}_{h}\left(\alpha \mid y, y^{\prime}\right)-\frac{1}{2 \alpha \varepsilon_{0} \varepsilon_{M}} \cdot e^{-\alpha\left|y-y^{\prime}\right|}\right]
\end{aligned}
$$

where

$$
\begin{aligned}
& A_{1}(\alpha)=\frac{\Gamma_{L}}{1-\Gamma_{L} \Gamma_{U} e^{-2 \alpha h_{M}}}-K-\delta \frac{e^{-2 \alpha d}}{e^{-2 \alpha H}-1} \\
& A_{2}(\alpha)=\frac{\Gamma_{L} \Gamma_{U} e^{-2 \alpha h_{M}}}{1-\Gamma_{L} \Gamma_{U} e^{-2 \alpha h_{M}}}+\delta \frac{e^{-2 \alpha H}}{e^{-2 \alpha H}-1} \\
& A_{3}(\alpha)=\frac{\Gamma_{U} e^{-2 \alpha h_{M}}}{1-\Gamma_{L} \Gamma_{U} e^{-2 \alpha h_{M}}}-\delta \frac{e^{-2 \alpha(H+d)}}{e^{-2 \alpha H}-1}
\end{aligned}
$$

being

$$
K=\frac{\varepsilon_{M}-\varepsilon_{M-1}}{\varepsilon_{M}+\varepsilon_{M-1}}
$$

In all the above expressions $\delta=1$ in the presence of a top ground plane, and $\delta=0$ if there is no top ground plane.

The first two terms at the RHS in (7) correspond to the source point and the first real image. The spectral functions $A_{i}(\alpha)\{i=1,2,3\}$ can be expanded as sums of complex exponentials. These terms can be viewed as the contributions of certain complex images, following the terminology in [20]. Usually just a few of these complex images are enough to get a very accurate representation of the Green's function. Therefore, after approximating the $A_{i}$ coefficients in (8) as short series of complex exponential functions with arguments $\left(-\alpha b_{n}\right)$ and amplitudes $B_{n}$, we obtain from (3) and (7) the following space-domain Green's function:

$$
\begin{aligned}
& G\left(x, y \mid x^{\prime}, y^{\prime}\right) \\
& =-\frac{1}{4 \pi \varepsilon_{0} \varepsilon_{M}}\left\{\ln \left[\left(x-x^{\prime}\right)^{2}+\left(y-y^{\prime}\right)^{2}\right]+\frac{\varepsilon_{M}-\varepsilon_{M-1}}{\varepsilon_{M}+\varepsilon_{M+1}}\right. \\
& \cdot \ln \left[\left(x-x^{\prime}\right)^{2}+\left(y+y^{\prime}\right)^{2}\right] \\
& \left.+\sum_{n=1}^{N_{c i}} B_{n} \ln \left[\left(x-x^{\prime}\right)^{2}+\left(y \pm y^{\prime} \pm b_{n}\right)^{2}\right]\right\} \\
& +\delta\left\{G_{h}\left(x, y \mid x^{\prime}, y^{\prime}\right)+\frac{1}{4 \pi \varepsilon_{0} \varepsilon_{M}}\right. \\
& \left.\cdot \ln \left[\left(x-x^{\prime}\right)^{2}+\left(y-y^{\prime}\right)^{2}\right]\right\}
\end{aligned}
$$

where $N_{c i}$ is the total number of employed complex images. Once again, the term affected by the factor $\delta$ in (9) is present only if we have a top ground plane. This term does not present a logarithmic singularity in the definition domain and, therefore, will not lead later to integration problems. Nevertheless, in the computer implementation of the code, we have extracted out not only the singular term, but also the first two real images (reflections on the top and bottom ground planes) in order to avoid lengthy integrations when the source and field points are very close to the ground plates.

In order to get some insight about the features of the approximation we are using, we include in this section some numerical examples. Thus, Fig. 2 shows the relative error when a typical spectral function-such as the ones found in our problem-is approximated by an increasing number of complex images. In this example, we have approximated 


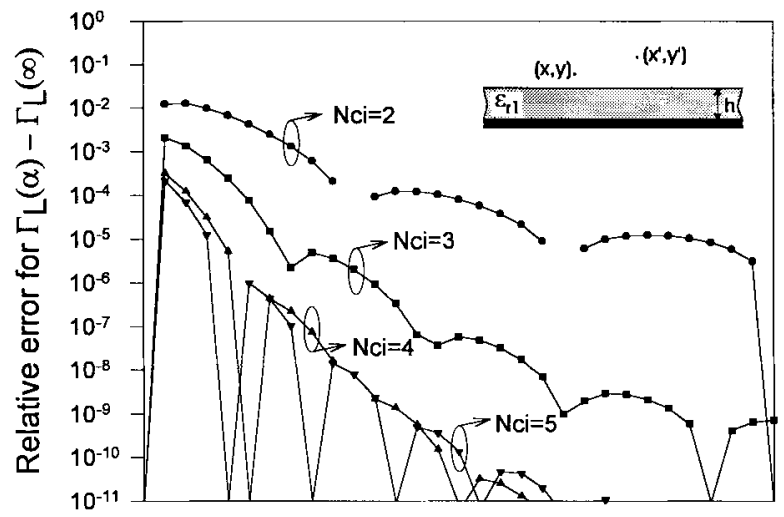

(a)

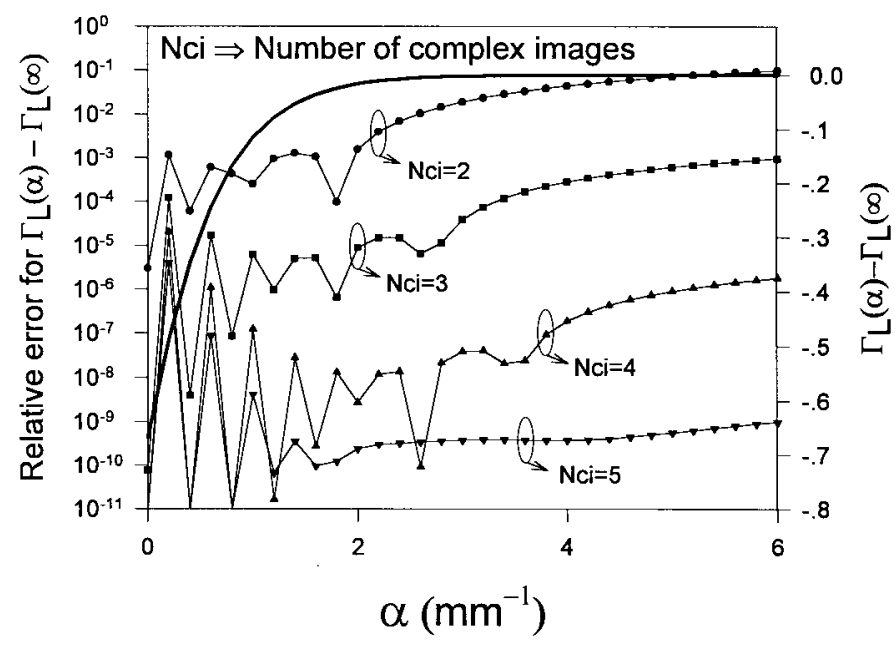

(b)

Fig. 2. Relative error in the approximation of a typical spectral function in our problem by using (a) Prony's method, and (b) MPM. We present curves for 2-5 complex images. $\Gamma_{L}(\alpha)$ corresponds to a single dielectric layer $\left(\varepsilon_{r}=2, h_{1}=1 \mathrm{~mm}\right)$ on a perfect ground plane.

$\Gamma_{L}(\alpha)-\Gamma_{L}(\infty)$, which is the sole function to be approximated when dealing with the standard microstrip structure. The complex images have been found by applying Prony's method [Fig. 2(a)] and the MPM [Fig. 2(b)]. From these figures we conclude that no more than four or five images are necessary to get a very good representation of the original Green's function in the spectral domain and, therefore, in the space domain. Fig. 2(b) also includes the function to be approximated. Notice that although the Prony approximation is more accurate when $\alpha$ increases, the use of the MPM seems to be advisable because this approach yields better results in the region where the approximated function is meaningfully different from zero. This feature has been confirmed for a large number of numerical examples. In brief, we conclude after a lot of numerical tests that just a few images computed with the MPM will ensure a high-quality approximation to the required space-domain Green's function.

\section{Application of the Galerkin Method}

In Section III, we have described a simple method to obtain an approximate closed-form expression for the space-domain
Green's function. Now we have to solve the integral equation for the surface free-charge distribution on the rectangular conductors by using the Galerkin method. It is well known that the efficiency of this technique depends to a large extent on the suitability of the chosen basis functions. In our particular case we should use functions accounting for the singular behavior at the metallic corners. Nevertheless, we have chosen a different criterion. We are more interested in using functions leading to closed-form formulas for the elements of the Galerkin matrix in order to speed up the filling of such a matrix. But, in addition, the number of functions needed to get a given accuracy should be kept as low as possible. These two goals can be achieved by using each of the faces of the rectangular conductors or the basis functions usually employed to approximate the charge distribution on zero thickness strips: first-kind Chebyshev polynomials weighed by the Maxwell distribution. In this way, for a given conducting face of width $w$ we write

$$
\sigma(t) \approx \frac{2}{\pi w} \sum_{n=0}^{n_{f}} C_{n} \frac{T_{n}\left(\frac{t-t_{0}}{w / 2}\right)}{\sqrt{1-\left(\frac{t-t_{0}}{w / 2}\right)^{2}}}
$$

where $t$ stands for the $x$ or $y$ variable (for horizontal and vertical conducting faces, respectively), $t_{0}$ is the middle point of the interval where the charge density is approximated, and $n_{f}$ is the number of retained basis functions on that face.

An important point is that the functions in (10) only partially account for the singularities at the corners. However, they allow us a quick filling of the Galerkin matrix. This is because some of the required integrations can be performed in closed form. When we use the Green's function in the form given in (9) and the basis functions in (10), we have to carry out the following integrations:

$$
\int_{t_{0} \rightarrow w / 2}^{t_{0}+w / 2} \ln \left[\left(x-x^{\prime}\right)^{2}+\left(y \pm y^{\prime} \pm b_{n}\right)^{2}\right] \cdot \frac{T_{p}\left(\frac{t^{\prime}-t_{0}}{w / 2}\right)}{\sqrt{1-\left(\frac{t^{\prime}-t_{0}}{w / 2}\right)^{2}}} d t^{\prime}
$$

where $t^{\prime}$ stands for $x^{\prime}$ or $y^{\prime}$. The integrals in (11) can be written in terms of the following one:

$$
\int_{-1}^{1} \ln \left(z_{1}+t z_{2}+j z_{3}\right) \frac{T_{p}(t)}{\sqrt{1-t^{2}}} d t=f\left(z_{1}, z_{2}, z_{3}\right) .
$$

This integral has a closed form and can be expressed as follows:

Case a) $(p>0)$ :

$$
f\left(z_{1}, z_{2}, z_{3}\right)=-\frac{\pi}{p}\left[\xi\left(z_{1}, z_{2}, z_{3}\right)\right]^{p}
$$

Case b) $(p=0)$ :

$$
f\left(z_{1}, z_{2}, z_{3}\right)=\pi \ln \left[\frac{-z_{2}}{2 \xi\left(z_{1}, z_{2}, z_{3}\right)}\right]
$$


where

$$
\xi\left(z_{1}, z_{2}, z_{3}\right)=-\frac{z_{1}+j z_{3}}{z_{2}} \pm \sqrt{\left(\frac{z_{1}+j z_{3}}{z_{2}}\right)^{2}-1} .
$$

(The sign before the square root is chosen in such a way that $|\xi|<1)$.

We must mention here that the real part of the integral in (12) was reported by Fikioris et al. in [1], and that we have also essentially used the same technique to get the imaginary part of (12).

The remaining integrations (inner products with the test functions) can now be carried out by using low-order Gauss-Chebyshev quadratures. If a top ground plane is present we have to still account for the contribution of the $\delta$ term in (9). This is easily done performing a double low-order Gauss-Chebyshev quadrature, owing to the mathematical nature of the integrand. The final result is that the Galerkin matrix is filled with low computational effort. This could also be done for simple subsectional pulse or triangular basis functions. The advantage of using the functions in (10) is that we do not need too many of them to get very accurate results, as will be shown in the Section V. Therefore, the Galerkinmatrix size will be small and the overall computation time will be low.

\section{NUMERICAL RESUltS}

As a first step in the analysis of the numerical behavior of the proposed technique, we have identified the different factors affecting the accuracy of the final results. In Section III, we said some words about the computation of the spacedomain Green's function. Since this function is numerically computed by means of an approximate method, its values will be affected by a certain error. However, this error can be systematically reduced by increasing the number of complex images. In fact, just a few of these images ensure a relative error well below one part in $10^{5}$ in the whole range of interest. Another source of error of numerical type can be found in the evaluation of the numerical quadratures needed to compute some integrals. After a lot of numerical experiments, we concluded that very good results will be obtained by using a number of quadrature points exceeding in two the order of the higher order Chebyshev polynomial used in the basis-function's expansion. In the case of inner products corresponding to functions defined on touching strip segments, this number should be increased to 10 . With this choice, we do not detect errors associated with erroneous computation of definite integrals.

However, the main factor affecting the accuracy of the final results is the number of basis functions retained in the expansion of the free-charge distribution. We are interested in getting good enough results with few basis functions so as to keep the size of the Galerkin matrix small. Obviously, our computer code permits us to consider the case of zero-thickness strips as a particular case. We have made comparisons of the results provided by our code with other practically exact results reported in the literature [1]-[3]. Our
TABLE I

$C_{11}(+)$ AND $C_{12}(-)(\mathrm{pF} / \mathrm{m})$ FOR THE STRUCtURe IN THE Figure Against the Number of Basis Functions on Vertical $\left(N_{t}\right)$ And Horizontal $\left(N_{w}\right)$ Conductor Faces. $w=1$, $s=t=0.2, h_{1}=3, h_{2}=2(\mathrm{~mm}) . \varepsilon_{r 1}=2.5, \varepsilon_{r 2}=10$

\begin{tabular}{c|c|c|c|c|c|c}
\multicolumn{2}{c}{} & \multicolumn{6}{c}{$N_{t}$} \\
\cline { 3 - 7 } \multicolumn{1}{c}{} & 1 & 2 & 3 & 4 & 5 \\
\hline \multirow{4}{*}{$N_{w}$} & 1 & 210.69 & 211.55 & 211.84 & 211.92 & 211.97 \\
& & -102.46 & -103.17 & -103.36 & -103.43 & -103.47 \\
\cline { 2 - 7 } & 2 & 216.70 & 216.85 & 217.11 & 217.12 & 217.14 \\
& & -107.60 & -107.75 & -107.93 & -107.94 & -107.96 \\
\cline { 2 - 7 } & 3 & 216.98 & 217.05 & 217.37 & 217.37 & 217.40 \\
& & -107.66 & -107.72 & -108.01 & -108.02 & -108.04 \\
\cline { 2 - 7 } & \multirow{2}{*}{4} & 217.01 & 217.06 & 217.38 & 217.39 & 217.42 \\
& & -107.68 & -107.73 & -108.03 & -108.03 & -108.06 \\
\cline { 2 - 7 } & \multirow{2}{*}{5} & 217.10 & 217.14 & 217.40 & 217.40 & 217.43 \\
& & -107.76 & -107.79 & -108.04 & -108.04 & -108.06 \\
\hline
\end{tabular}

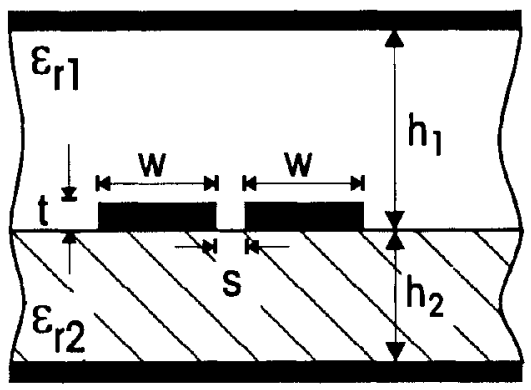

program also yields virtually exact results because for the zero thickness case, it is a quasi-analytical microstrip solver (such as the methods reported in the cited papers), with extremely low central processing unit (CPU) time consumption. This excellent performance is related to the nature of the used basis functions-which are especially suitable for zero thickness strips-and to the analytical treatment of the computation of the Galerkin matrix entries. Therefore, as a subproduct of our analysis, we have an extremely efficient code for the analysis of zero thickness coupled strips available. Nevertheless, in the context of this paper, we are more interested in the case of nonzero thickness strips. Therefore, we show in Table I the convergence of the capacitance coefficients for a pair of thick coupled microstrips. From Table I, we can see that convergence is not so good as the one we achieved in the analysis of zero-thickness coupled strips [2]. The reason for this is that the employed basis functions do not exactly fit the corner behavior. However, these functions are very good from a practical point of view: two basis functions per strip side yield results with an accuracy better than $0.3 \%$. Of course, the number of basis functions has to be increased when the geometry is more critical (for example, when coupling between the strips is extremely strong or in other geometrically complicated situations). Nevertheless, we usually obtain very good results with just a few basis functions per strip side. Getting similar accuracy with subsectional-type basis functions would require many more basis functions, therefore increasing the CPU time.

After analyzing the convergence of the method, it is interesting to check the typical attainable accuracy using some 
TABLE II

$Z_{0}$ of a Symmetrical Shielded Slab Line $N_{w, t}$ IS THE Number of Basis Functions $W=$ SLAB Width. $t=$ SLAB

Thickness $b=$ Separation Between Ground Plates

\begin{tabular}{c|c|c|c|c|c}
\hline $\mathrm{W} /(\mathrm{b}-\mathrm{t})$ & $\mathrm{t} / \mathrm{b}$ & Exact [25] & $N_{w, t}=8$ & $N_{w, t}=3$ & $N_{w, t}=1$ \\
\hline 0.1 & 0.0 & 194.226 & 194.226 & 194.226 & 194.226 \\
0.1 & 0.1 & 145.665 & 145.665 & 145.676 & 146.137 \\
0.1 & 0.5 & 83.262 & 83.261 & 83.270 & 83.413 \\
0.1 & 0.9 & 43.079 & 43.074 & 43.085 & 43.679 \\
\hline 0.2 & 0.0 & 153.029 & 153.029 & 153.029 & 153.030 \\
0.2 & 0.1 & 123.293 & 123.293 & 123.304 & 123.747 \\
0.2 & 0.5 & 75.928 & 75.928 & 75.937 & 76.165 \\
0.2 & 0.9 & 41.054 & 41.050 & 41.063 & 41.529 \\
\hline
\end{tabular}

appropriate benchmark. We can use as a benchmark the rectangular slab symmetrically placed between two infinite ground planes, since for this structure there exists an analytical solution. Table II shows the comparison between exact solutions taken from [25] (conformal mapping approach) and numerical values computed with our method. It is clear from this table that our method can provide very high accuracy if we use enough basis functions. However, even using just one function on each side of the slab, we get results within a $0.4 \%$ error except for the worst case $(t / b=0.9)$, where the error is around $1.5 \%$. In this case, using just one more function on the long sides of the slab results in a drastic improvement of the accuracy (less than $0.02 \%$ error). Other comparisons have been made with some exact results reported in [26] with similar conclusions. Convergence to the correct value has then been demonstrated. Note that this point is important since our basis functions do not exhibit the rigorously correct edge behavior.

In [19], the author presents a technique based on spectraldomain analysis (SDA) to compute the quasi-static parameters of boxed coupled strips of arbitrary thickness. We have made comparisons with the results reported in [19] and we have found very good agreement. In particular, we have computed the modal parameters of two very tightly coupled strips, which cannot be efficiently treated by using the simple multistrip model reported in [15] (as was claimed in [19]). Our results are very accurate for this case even using only two basis functions on the faced sides of the strips and one on the remaining sides ( $0.2 \%$ error). This results in CPU times much shorter than the ones reported in [19] (ours is below $1 \mathrm{~s}$ on a $66-\mathrm{MHz}$ pentium-based PC).

Our program can be used to calculate the resistance matrix $[R]$ of the multiconductor system just applying the Wheeler's rule, such as discussed in [21]:

$$
[R]=\frac{R_{s}}{\mu_{0}} \frac{\partial[L]}{\partial \xi}
$$

where $R_{s}$ is the surface resistance of the metal, [L] is the inductance matrix, and $\xi$ is the coordinate variable normal to the conductor surface. The derivative is numerically computed, so we only need to accurately compute [L]. Table III shows the normalized resistance for a rectangular slab on a ground
TABLE III

Normalized Resistance of Rectangular Slab Over Ground Plane as a Function of the Number of Basis Functions $(n f)$ on Each StRip Side AND of the $\Delta \xi$ InCREMENT Used in the Numerical Derivation Slab Width $=2 a$, Slab Thickness $=a$, Distance to Ground Plane $=a$

\begin{tabular}{c|c|c|c|c}
\cline { 2 - 5 } \multicolumn{2}{c}{$a R / R_{s}$} \\
\cline { 2 - 5 } $\mathrm{nf}$ & $\Delta \xi=a \times 10^{-1}$ & $a \times 10^{-2}$ & $a \times 10^{-3}$ & $a \times 10^{-4}$ \\
\hline 1 & 0.23541 & 0.23394 & 0.23392 & 0.23392 \\
\hline 2 & 0.23542 & 0.23395 & 0.23393 & 0.23393 \\
\hline 3 & 0.23757 & 0.23607 & 0.23605 & 0.23605 \\
\hline 4 & 0.23758 & 0.23608 & 0.23607 & 0.23607 \\
\hline 5 & 0.23758 & 0.23608 & 0.23606 & 0.23606 \\
\hline
\end{tabular}

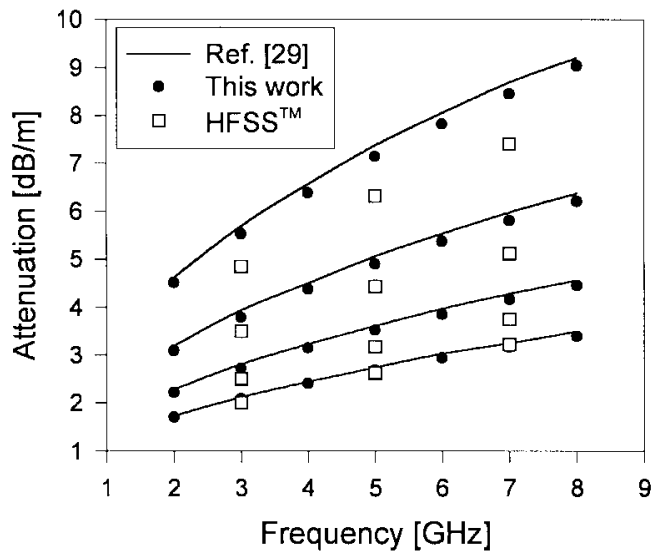

Fig. 3. Attenuation factors for the fundamental quasi-TEM modes of the four microstrips system analyzed in [27]. Strip widths: $w_{1}=w_{4}=0.6$ $\mathrm{mm}, w_{2}=w_{3}=0.3 \mathrm{~mm}$, separation between strips: $s_{1}=s_{3}=0.3 \mathrm{~mm}$, $s_{2}=0.2 \mathrm{~mm}$, strip thickness: $t=0.01 \mathrm{~mm}$, substrate thickness: $h=0.635$ $\mathrm{mm}$, height to cover: $d=6.0 \mathrm{~mm}, \varepsilon=9.8 \varepsilon_{0}, \sigma=5.1 \cdot 10^{7} \mathrm{~S} / \mathrm{m}$

plane as a function of the number of basis functions used on each strip side. The result is close to the one reported in [13] $\left(R / R_{s}=0.23753\right)$. We have included several columns to show how the $\Delta \xi$ increment used to numerically perform the derivation affects the final result. We can typically choose $\Delta \xi$ around $10^{-3}$ times the smallest dimension of the conductor. The result remains stable for lower values of $\Delta \xi$. Note that the derivative is very accurately computed even though the original function is affected by a certain error, since this is a systematic error that affects in the same magnitude the two values of the function required to numerically perform the derivation. Recently, Gentili et al. [27] have reported a technique to compute the modal attenuation factors $\left(\alpha_{k} k=\right.$ $1, \cdots, N_{c}$ ) for a multiconductor line. The values of the $\alpha_{k}$ parameters can be readily obtained from the characteristic matrices computed in this paper. This way, we have compared our results with those reported in [27], with very good agreement. For instance, we show in Fig. 3 the four modal attenuation factors corresponding to the fundamental modes supported by a four-coupled-strip lossy system. Our results are very close to the ones computed by the method proposed by Gentili et al. (slight differences could be attributed to the side walls considered in that paper), but some important discrepancies have been found when comparing the results computed by means of a finite-element-based code. 
TABLE IV

Coefficients of [C], [L], And [R] for the Five Conductors' Structure in the Figure. $w=3, s=2, h=1, t=1(\mathrm{~mm}), \varepsilon_{r} 1=2, \varepsilon_{r 2}=1$.

\begin{tabular}{c|c|c|c|l}
\hline $\mathrm{i}$ & $\mathrm{j}$ & $C_{i j}(\mathrm{pF} / \mathrm{m})$ & $L_{i j}(\mu \mathrm{H} / \mathrm{m})$ & \multicolumn{1}{|c}{$R / R_{s}$} \\
\hline 1 & 1 & 93.668 & $19.781 \times 10^{-2}$ & 0.1885 \\
1 & 2 & -8.453 & $2.946 \times 10^{-2}$ & $0.1347 \times 10^{-1}$ \\
1 & 3 & -0.809 & $0.735 \times 10^{-2}$ & $0.4600 \times 10^{-2}$ \\
1 & 4 & -0.345 & $0.284 \times 10^{-2}$ & $0.2657 \times 10^{-2}$ \\
1 & 5 & -0.215 & $0.152 \times 10^{-2}$ & $0.1605 \times 10^{-2}$ \\
2 & 2 & 95.329 & $19.471 \times 10^{-2}$ & 0.1939 \\
2 & 3 & -8.318 & $2.883 \times 10^{-2}$ & $0.1459 \times 10^{-1}$ \\
2 & 4 & -0.758 & $0.717 \times 10^{-2}$ & $0.4879 \times 10^{-2}$ \\
3 & 3 & 95.341 & $19.458 \times 10^{-2}$ & 0.1941 \\
\hline
\end{tabular}

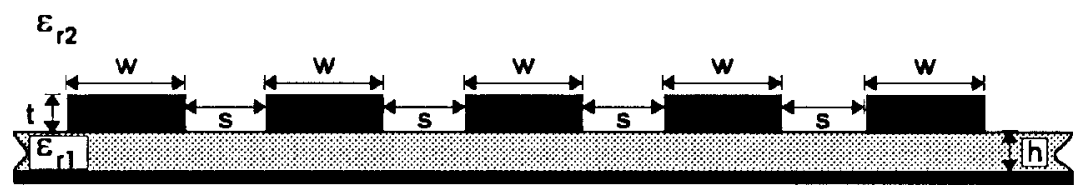

Finally, Table IV shows the elements of $[\mathrm{C}]$ and $[\mathrm{L}]$ for a five-coupled-strip system. All the figures in Table IV are correct. We have used 15 basis functions on each strip side, i.e., a total of 300 basis functions, to ensure the accuracy of these results. This results in a computation time of $75 \mathrm{~s}$ on a Pentium PC/66 MHz. However, accuracy better than $0.2 \%$ for all the matrix elements is achieved by using five functions and $13 \mathrm{~s}$ of CPU time. If an error in the order of $1 \%$ can be tolerated, we only have to use two functions per strip side. We have completed Table IV, including the normalized resistance matrix elements.

\section{CONCLUSIONS}

This paper describes an efficient and accurate technique to compute the quasi-static matrix parameters $([\mathrm{C}],[\mathrm{L}],[\mathrm{R}]$, [G]) of a system of rectangular cross-section coupled conductors embedded in a layered dielectric medium. Accuracy and numerical efficiency are achieved by means of two main issues. First, we use entire domain basis functions to approximate the free surface-charge distribution on the strips. These functions allow us to keep the Galerkin matrix size small when compared with typical matrix size associated with the use of subsectional functions. Second, we get quasi-analytical evaluation of the integrals defining the Galerkin-matrix entries. This has been done by taking advantage of the use of the complex image concept and the mathematical properties of the employed basis functions. Any spectral estimation technique can be used to compute the complex images, but the MPM seems to perform better than Prony's method. The final product is an accurate and quick computer code that permits one to analyze under quasiTEM assumption a variety of transmission lines consisting of coupled rectangular conducting slabs. The performance of the code has been exhaustively checked by making convergence tests and comparisons with other techniques, some of which have been included in Section V. Note that although in this paper it has been assumed that all the strips were embedded in the same dielectric region, a more general situation can be treated by applying the same general ideas reported here.

\section{REFERENCES}

[1] J. G. Fikioris, J. L. Tsalamengas, G. J. Fikioris, "Exact solutions for shielded printed microstrip lines by the Carleman-Vekua method," IEEE Trans. Microwave Theory Tech., vol. 37, pp. 21-33, Jan. 1989.

[2] E. Drake, F. Medina, M. Horno, "Improved quasi-TEM spectral domain analysis of boxed coplanar multiconductor microstrip lines," IEEE Trans. Microwave Theory Tech., vol. 41, pp. 260-267, Feb. 1993.

[3] D. Homentcovschi, G. Ghione, C. Naldi, R. Oprea, "Analytic determination of the capacitance matrix of planar or cylindrical multiconductor lines on multilayered substrates," IEEE Trans. Microwave Theory Tech., vol. 43, pp. 363-372, Feb. 1995.

[4] H. E. Green, "The numerical solution of some important transmissionline problems," IEEE Trans. Microwave Theory Tech., vol. MTT-13, pp. 676-692, Sept. 1965.

[5] Z. Pantic and R. Mittra, "Quasi-TEM analysis of microwave transmission lines by the finite-element method," IEEE Trans. Microwave Theory Tech., vol. MTT-34, pp. 1096-1103, Nov. 1986.

[6] J. M. Gil and J. Zapata, "Efficient singular element for finite element analysis of quasi-TEM transmission lines and waveguides with sharp metal edges," IEEE Trans. Microwave Theory Tech., vol. 42, pp. 92-98, Jan. 1994.

[7] Z. Pantic and R. Mittra, "Higher-order infinite elements for quasiTEM analysis of microwave transmission lines using the finite-element method," Microwave Opt. Technol. Lett., vol. 9, no. 5, pp. 244-249, Aug. 1995.

[8] A. Papachristoforos, "Method of lines for analysis of planar conductors with finite thickness," Proc. Inst. Elect. Eng.-Microwaves, Antennas, Propagat., vol. 141, no. 3, pp. 223-228, June 1994.

[9] C. Wei, R. F. Harrington, J. R. Mautz, and T. K. Sarkar, "Multiconductor transmission lines in multilayered dielectric media," IEEE Trans. Microwave Theory Tech., vol. MTT-32, pp. 439-450, Apr. 1984.

[10] J. Venkataraman, S. M. Rao, A. R. Djordjevic, T. K. Sarkar, and Y. Naiheng, "Analysis of arbitrarily oriented microstrip transmission lines in arbitrarily shaped dielectric media over a finite ground plane," IEEE Trans. Microwave Theory Tech., vol. MTT-33, pp. 952-959, Oct. 1985.

[11] M. B. Bazdar, A. R. Djordjevic, R. F. Harrington, and T. K. Sarkar, "Evaluation of quasi-static matrix parameters for multiconductor transmission lines using Galerkin's method," IEEE Trans. Microwave Theory Tech., vol. 42, pp. 1223-1228, July 1994.

[12] W. Delbare and D. De Zutter, "Space-domain Green's function approach to the capacitance calculation of multiconductor lines in multilayered dielectrics with improved surface charge modeling," IEEE Trans. Microwave Theory Tech., vol. 37, pp. 1562-1568, Oct. 1989.

[13] F. Olyslager, N. Faché, and D. de Zutter, "New fast and accurate line parameter calculation of general multiconductor transmission lines in multilayered media," IEEE Trans. Microwave Theory Tech., vol. 39, pp. 901-909, June 1991. 
[14] K. S. Oh, D. Kuznetsov, and J. E. Schutt-Aine, "Capacitance computations in a multilayered dielectric medium using closed-form spatial Green's functions," IEEE Trans. Microwave Theory Tech., vol. 42, pp. 1443-1453, Aug. 1994.

[15] G. Plaza, F. Mesa, and M. Horno, "Quick computation of [C], [L], $[\mathrm{G}]$, and $[\mathrm{R}]$ matrices of multiconductor and multilayered transmission systems," IEEE Trans. Microwave Theory Tech., vol. 43, pp. 1623-1626, July 1995.

[16] V. Rizzoli, "Highly efficient calculation of shielded microstrip structures in the presence of undercutting," IEEE Trans. Microwave Theory Tech. vol. MTT-27, pp. 150-157, Feb. 1979.

[17] E. Drake, F. Medina, and M. Horno, "Quasi-TEM analysis of thick multistrip lines using an efficient iterative method," Microwave Opt. Technol. Lett., vol. 5, no. 10, pp. 530-534, Sept. 1992

[18] G. G. Gentili and G. Macchiarella, "Quasi-static analysis of shielded planar transmission lines with finite metallization thickness by a mixed spectral-space domain method," IEEE Trans. Microwave Theory Tech., vol. 42, pp. 249-255, Feb. 1994.

[19] J.-T. Kuo, "Accurate quasi-TEM spectral domain analysis of single and multiple coupled microstrip lines of arbitrary metallization thickness," IEEE Trans. Microwave Theory Tech., vol. 43, pp. 1881-1888, Aug. 1995.

[20] Y. L. Chow, J. J. Yang, and G. E. Howard, "Complex images for electrostatic field computation in multilayered media," IEEE Trans. Microwave Theory Tech., vol. 39, pp. 1120-1125, July 1991.

[21] G. Plaza, F. Mesa, and M. Horno, "Spectral domain analysis of conductor losses in a multiconductor system via the incremental inductance rule," Electron. Lett., vol. 30, no. 17, pp. 1425-1427, Aug. 1994.

[22] R. Crampagne, M. Ahmadpanah, and J.-L. Guiraud, "A simple method for determining the Green's function for a large class of MIC lines having multilayered dielectric substrates," IEEE Trans. Microwave Theory Tech., vol. MTT-26, pp. 82-87, Feb. 1978.

[23] F. Medina and M. Horno, "Upper and lower bounds on mode capacitances for a large class of anisotropic multilayered microstrip-like transmission lines," Proc. Inst. Elect. Eng.-Microwaves, Antennas, Propagat., vol. 132, pt. H, no. 3, pp. 157-163, June 1985.

[24] T. K. Sarkar and O. Pereira, "Using the matrix pencil method to estimate the parameters of a sum of complex exponentials," Antennas Propagat. Mag., vol. 37, no. 1, pp. 48-55, Feb. 1995.

[25] H. J. Riblet, "An approximation for the characteristic impedance of shielded-slab lines," IEEE Trans. Microwave Theory Tech., vol. MTT-27, pp. 557-559, June 1979 .

[26] J. R. Nortier, "Calculation of the interaction between the fringing capacitance of symmetrical stripline using the finite element method," IEEE Trans. Microwave Theory Tech., vol. MTT-34, pp. 191-193, Jan. 1986.

[27] G. G. Gentili and A. Melloni, "The incremental inductance rule in quasiTEM coupled transmission lines," IEEE Trans. Microwave Theory Tech. vol. 43, pp. 1276-1280, June 1995.

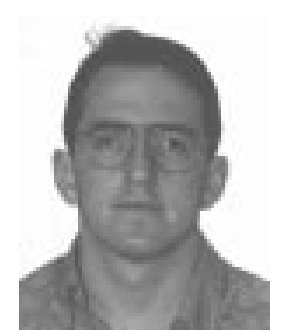

Joaquín Bernal was born in Sevilla, Spain, in 1971. He received the Licenciado degree in physics from the University of Seville, Spain, in 1994, and is currently working toward the Ph.D. degree.

His research interests focus on the analysis of planar structures for integrated microwave circuits.

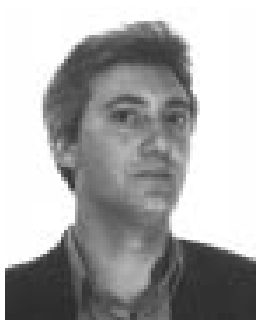

Francisco Medina was born in Puerto Real, Cádiz, Spain, in November, 1960. He received the Licenciado and the Doctor degrees, both in physics, from the University of Seville, Seville, Spain, in 1983 and 1987, respectively. From 1986 to 1987, he spent the academic year at the Laboratoire de Microondes de l'ENSEEIHT, Toulouse, France, on scholarship from MEC-MRT.

From 1985 to 1989 , he was an Assistant Professor in the Department of Electronics and Electromagnetics, University of Seville, and since 1990, he has been a Profesor Titular (Associate Professor) of electromagnetics. His research deals mainly with analytical and numerical methods for planar structures and circuit applications of multiconductor lines.

Dr. Medina was a member of the Technical Programme Committee of the 23rd European Microwave Conference, Madrid, Spain, in 1993.

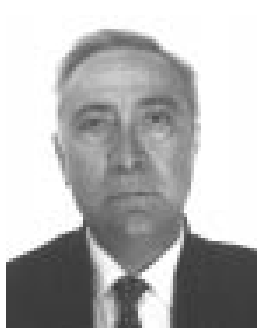

Manuel Horno (M'75) was born in Torre del Campo, Jaén, Spain. He received the Licenciado and the Doctor degrees, both in physics, from the University of Seville, Seville, Spain, in 1969, and 1972, respectively.

Since 1969, he has been with the Department of Electronics and Electromagnetism, University of Seville, where he became an Assistant Professor in 1970, Associate Professor in 1975, and Full Professor in 1986. His main fields of interest include boundary value problems in electromagnetic theory, wave propagation through anisotropic media, and microwave integrated circuits. He is presently engaged in the analysis of planar transmission lines embedded in complex materials, multiconductor transmission lines, and printed antennas.

Dr. Horno is a member of the Electromagnetism Academy, Massachusetts Institute of Technology (MIT), Cambridge. 